Astronomy Letters, 2010 Vol. 36, No. 3

\title{
Searching for Stars Closely Encountering with the Solar System
}

\author{
V.V. Bobylev \\ Pulkovo Astronomical Observatory, Russian Academy of Sciences, St-Petersburg
}

\begin{abstract}
Based on a new version of the Hipparcos catalog and currently available radial velocity data, we have searched for stars that either have encountered or will encounter the solar neighborhood within less than $3 \mathrm{pc}$ in the time interval from $-2 \mathrm{Myr}$ to $+2 \mathrm{Myr}$. Nine new candidates within 30 pc of the Sun have been found. To construct the stellar orbits relative to the solar orbit, we have used the epicyclic approximation. We show that, given the errors in the observational data, the probability that the well-known star HIP 89825 (GL 710) encountering with the Sun most closely falls into the Oort cloud is 0.86 in the time interval $1.45 \pm 0.06 \mathrm{Myr}$. This star also has a nonzero probability, $1 \times 10^{-4}$, of falling into the region $d<1000 \mathrm{AU}$, where its influence on Kuiper Belt objects becomes possible.
\end{abstract}

DOI:10.1134/S1063773710030060

\section{INTRODUCTION}

Interest in the problem of close encounters of field stars with the Solar system stems from the fact that the passage of a star can lead to various kinds of perturbations of Solar system objects.

Thus, for example, the interaction of a star with the Oort comet cloud can give rise to comet showers reaching the region of the major planets (Hills 1981; Weissman 1996). The formation time scale of a comet shower is $\approx 1$ Myr after the passage of a star. According to Weissman (1996), the Oort cloud is a spheroid with a semimajor axis of about $10^{5} \mathrm{AU}$ $(\approx 0.5 \mathrm{pc}$, since $1 \mathrm{pc}=206265 \mathrm{AU})$ elongated toward the Galactic center and a semiminor axis of about $8 \times 10^{4}$ AU. The question about close encounters of stars with the Sun within $r<2-5$ pc was considered by Revina (1988), Matthews (1994), and Mullari and Orlov (1996) using various ground-based observations and by Garcia-Sanchez et al. (1999, 2001) based on Hipparcos (1997) data in combination with stellar radial velocity data. As a result, 156 Hipparcos stars from the solar neighborhood 50 pc in radius that either have encountered or will encounter with the Solar system within $r<5 \mathrm{pc}$ in the time interval \pm 10 Myr are known to date (Garcia-Sanchez et al. 2001). Having analyzed these data, Garcia-Sanchez et al. (2001) estimated the frequency of close (within 1 pc) encounters of stars with the Sun to be $2.3 \pm 0.2$ encounters per Myr and, after a correction for the Hipparcos incompleteness, this value increases to $11.7 \pm 1.3$ encounters per Myr.

Analyzing the possibility of even closer encounters is of current interest. For example, Kenyon and Bromley (2004) argue that only the passage of a star can explain the peculiarities of the orbit of the minor planet 2003 VB12 (Sedna). The various kinds of influences of such a star on Kuiper Belt objects were shown to manifest themselves at characteristic distances 
$r<1000 \mathrm{AU}(\approx 0.005 \mathrm{pc})$. The Kuiper Belt proper extends from the Sun to a boundary of $\approx 50 \mathrm{AU}$.

The goal of this study is to search for candidate stars closely encountering with the Sun based on a new version of the Hipparcos catalog (van Leeuwen, 2007) and currently available stellar radial velocity data. We solve the problem of statistical simulations by taking into account the random errors in the input data and estimate the probability of a star penetrating into the Oort cloud region and into the region of a possible influence of the star on Kuiper Belt objects.

\section{THE DATA}

We use stars from the Hipparcos catalog (ESA 1997) while taking the new proper motions and parallaxes from a revised version of this catalog (van Leeuwen, 2007); the stellar radial velocities are taken from the Pulkovo Compilation of Radial Velocities (PCRV) (Gontcharov 2006) created as a result of implementing the OSACA project (Bobylev et al., 2006) and containing radial velocity data for about 35000 stars. Note that, in contrast to the larger CRVAD-2 catalog of radial velocities (Kharchenko et al., 2007), the PCRV catalog contains only the stars with random errors in their radial velocities within $10 \mathrm{~km} \mathrm{~s}^{-1}$.

\section{THE METHODS}

\subsection{Orbit Construction}

We use a rectangular Galactic coordinate system with the axes directed away from the observer toward the Galactic center $\left(l=0^{\circ}, b=0^{\circ}\right.$, the $X$ axis), in the direction of Galactic rotation $\left(l=90^{\circ}, b=0^{\circ}\right.$, the $Y$ axis), and toward the North Pole $\left(b=90^{\circ}\right.$, the $Z$ axis). The corresponding space velocity components of an object $U, V, W$ are also directed along the $X, Y, Z$ axes. The epicyclic approximation (Lindblad, 1927) allows the stellar orbits to be constructed in a coordinate system rotating around the Galactic center. We apply this method in the form given in Fuchs et al. (2006):

$$
\begin{gathered}
X(t)=X(0)+\frac{U(0)}{\kappa} \sin (\kappa t)+ \\
+\frac{V(0)}{2 B}(1-\cos (\kappa t)), \\
Y(t)=Y(0)+2 A\left(X(0)+\frac{V(0)}{2 B}\right) t- \\
-\frac{\Omega_{0}}{B \kappa} V(0) \sin (\kappa t)+\frac{2 \Omega_{0}}{\kappa^{2}} U(0)(1-\cos (\kappa t)), \\
Z(t)=\frac{W(0)}{\nu} \sin (\nu t)+Z(0) \cos (\nu t),
\end{gathered}
$$

where $t$ is the time in Myr (we proceed from the ratio pc/Myr $=0.978 \mathrm{~km} \mathrm{~s}^{-1}$ ); $A$ and $B$ are the Oort constants; $\kappa=\sqrt{-4 \Omega_{0} B}$ is the epicyclic frequency; $\Omega_{0}$ is the angular velocity of Galactic rotation for the local standard of rest, $\Omega_{0}=A-B ; \nu=\sqrt{4 \pi G \rho_{0}}$ is the vertical oscillation frequency, where $G$ is the gravitational constant and $\rho_{0}$ is the star density in the solar neighborhood. 
The parameters $X(0), Y(0), Z(0)$ and $U(0), V(0), W(0)$ in the system of equations (1) denote the current stellar positions and velocities. The displacement of the Sun from the Galactic plane is taken to be $Z(0)=17 \mathrm{pc}$ (Joshi 2007). We calculate the velocities $U, V, W$ relative to the local standard of rest $(\mathrm{LSR})$ with $(U, V, W)_{L S R}=(10.00,5.25,7.17) \mathrm{km} \mathrm{s}^{-1}$ (Dehnen and Binney 1998).

We took $\rho_{0}=0.1 M_{\odot} / \mathrm{pc}^{3}$ (Holmberg and Flinn 2004), which gives $\nu=74 \mathrm{~km} \mathrm{~s}^{-1} \mathrm{kpc}^{-1}$. We used the following Oort constants found by Bobylev et al. (2008): $A=15.5 \pm 0.3 \mathrm{~km}$ $\mathrm{s}^{-1} \mathrm{kpc}^{-1}$ and $B=-12.2 \pm 0.7 \mathrm{~km} \mathrm{~s}^{-1} \mathrm{kpc}^{-1} ; \kappa=37 \mathrm{~km} \mathrm{~s}^{-1} \mathrm{kpc}^{-1}$ corresponds to these values.

Note that we neglect the gravitational interaction between the star and the Sun.

As was shown by Mullari and Orlov (1996), even the simplest linear approximation provides a sufficient accuracy in the time interval from $-2 \mathrm{Myr}$ to $+2 \mathrm{Myr}$ compared to the method of constructing the relative orbits (the star relative to the Sun) in the Galactic potential. We choose the epicyclic approximation, because we are planning to perform statistical simulations with the computation of hundreds of thousands of orbits, while the application of more complex methods requires a huge computational time.

\subsection{Statistical Simulations}

In accordance with the method of Monte Carlo statistical simulations, we compute a set of orbits for each object by taking into account the random errors in the input data. For each star, we compute the encounter parameter, the shortest distance between the stellar and

solar orbits $d_{\text {min }}=\sqrt{\Delta X^{2}(t)+\Delta Y^{2}(t)+\Delta Z^{2}(t)}$. The stellar parameters are assumed to be distributed normally with a dispersion $\sigma$. We add the errors to the equatorial coordinates, proper motion components, parallax, and radial velocity of the star using the nominal errors.

\section{$3 \quad$ RESULTS}

First, we considered the solar neighborhood $30 \mathrm{pc}$ in radius using $\approx 35000$ stars with known space velocities. For each star, we constructed the orbit relative to the Sun in the time interval from -2 Myr to +2 Myr. In addition to the stars from the list by Garcia-Sanchez et al. (2001), we found several other Hipparcos stars. Data on the nine stars found with an encounter parameter $d<3$ pc are presented in Table 1; Fig. 1a shows the trajectories for six of these stars.

Figure $1 \mathrm{~b}$ shows the model trajectories of four stars computed by taking into account the random errors in the observational data; 300 realizations were obtained for each of the three stars indicated in the figure.

Table 2 gives characteristics of the stars common to the list by Garcia-Sanchez et al. (2001) that have the closest $(d<2$ pc) encounters with the Sun.

Table 3 gives characteristics of the common list of stars that have the closest $(d<$ $2 \mathrm{pc}$ ) encounters with the Sun. Note that this is currently the most complete list with the restrictions that we used. In contrast to Tables 1 and 2, Table 3 gives the encounter parameters $d_{\min }$ and $t_{\min }$ with their errors estimated through statistical simulations.

Table 3 also contains two stars from Table 1. The first of them is the well-known white dwarf WD 0310.688 (HIP 14754). Note that the error in its radial velocity (Pauli et al. 2006) is largest among all of the stars listed in Tables 1 and 2. The data for the second 
star, HIP 27887, are very reliable. It is contained in the catalog of F and G dwarfs of the Geneva-Copenhagen survey (Nordstrom et al. 2004; Holmberg et al. 2007).

Note that the compilations by Gontcharov (2006) and Kharchenko et al. (2007) give an obsolete radial velocity for HIP $3829, V_{r}=263 \pm 5 \mathrm{~km} \mathrm{~s}^{-1}$, taken from the GCRV (Wilson 1953). A very close encounter with the Sun is obtained with this value: $d_{\min }=0.96 \mathrm{pc}$ at $t_{\text {min }}=-16$ thousand years. However, this star is the well-known white dwarf WD $0046+051$ (van Maanens star, vMa 2). According to more recent observations, its radial velocity is $V_{r}=54 \mathrm{~km} \mathrm{~s}^{-1}$ (Greenstein and Trimble 1967) or $V_{r}(L S R)=-41 \mathrm{~km} \mathrm{~s}^{-1}$ (Aannestad et al. 1993). In this case, the encounter is considerably less close, $d_{\min }>3 \mathrm{pc}$ (see also Garcia-Sanchez et al. 1999, 2001).

Figure 2 shows the trajectories of HIP 89825 (GL 710) relative to the Sun computed by taking into account the random errors in the observational data.

We made only 300 realizations for a clearer graphical presentation. It is obvious from the figure that very close encounters with the Solar system are possible for this star. More detailed simulations show that, for example, out of the one million model orbits found for GL $710, d_{\text {min }} \leq 0.48 \mathrm{pc}$ in 855902 cases and, hence, the probability of its falling into the Oort cloud is $P_{1}=0.86$ and $d_{\text {min }} \leq 0.005$ pc in 94 cases and, hence, the probability of the star approaching the boundary of its possible influence on Kuiper Belt objects is $P_{2}=0.94 \times 10^{-4}$.

Figure 3 show the distribution of encounter parameters of HIP 89825 (GL 710) with the Sun obtained using one million model orbits.

\section{DISCUSSION}

(1) Among the Hipparcos stars we consider, there are nine stars common to the list by Mullari and Orlov (1996). The encounter parameters for some of the stars found by Mullari and Orlov $(1996)$ are: $d_{\text {min }}=1.35 \mathrm{pc}, t_{\min }=1050$ thousand years for GL $710 ; d_{\min }=1.64$ $\mathrm{pc}, t_{\min }=-529$ thousand years for GL 208 ; and $d_{\min }=1.89 \mathrm{pc}, t_{\min }=88.7$ thousand years for GL $860 \mathrm{~A}$. The encounter parameters agree well with our results (Table2). There is only one exception, GL 710, for which the new data give a closer encounter, which was also pointed out by Garcia-Sanchez et al. (1999).

Note that the star HIP 99461 (GL 783) we selected is present in the list by Mullari and Orlov (1996) but is absent in the lists by Garcia-Sanchez et al. (1999, 2001).

(2) For an overwhelming majority of the stars, the encounter parameters derived here agree well with those from Garcia-Sanchez et al. (2001).

The differences are most noticeable for the star GL 217.1, more specifically, $\left|\Delta d_{\text {min }}\right|=$ $0.38 \mathrm{pc}$ and $\left|\Delta t_{\min }\right|=182$ thousand years. According to Garcia-Sanchez et al. (2001), who adopted the radial velocity $V_{r}=20.0 \pm 3.7 \mathrm{~km} \mathrm{~s}^{-1}$ that differs markedly from our value (Table 2), the encounter parameters for GL 217.1 are: $d_{\min }=1.65 \pm 0.27 \mathrm{pc}$ and $t_{\text {min }}=-(1046 \pm 163)$ thousand years.

Computing the model epicyclic orbits for the star GL 217.1 using the initial data from Garcia-Sanchez et al. (2001) with the adopted radial velocity error $e_{V_{r}}=2 \mathrm{~km} \mathrm{~s}^{-1}$ yields $d_{\text {min }}=1.61 \pm 0.20 \mathrm{pc}$ and $t_{\min }=-(1066 \pm 116)$ thousand years, and these parameters almost coincide with those from Garcia-Sanchez et al. (2001).

This leads us to conclude that the existing discrepancy is undoubtedly related to the difference in input data.

As we see from Table 3, GL 217.1 is the most massive, $M=2 M_{\odot}$, star among our stars. 
Its improved encounter parameters, $d_{\min }=1.28 \pm 0.06 \mathrm{pc}$ and $t_{\min }=-(861 \pm 40)$ thousand years, make it attractive for studying the close passages that could take place in the past.

(3) The star GL 710 is of greatest interest to us, because it has a record close encounter with the Sun among all of the candidates known to date. According to Garcia-Sanchez et al. (2001), the encounter parameters of GL 710 are: $d_{\text {min }}=0.34 \pm 0.18$ pc and $t_{\text {min }}=(1358 \pm 41)$ thousand years, in good agreement with those we found (Table 3).

GL 710 is the only star with a significant difference between the data of Tables 2 and 3. This suggests that the conditions for its encounter with the Sun make the stellar orbit very sensitive to small changes in such initial data as the parallax and radial velocity. We clearly see from Fig. 3a that the distribution of minimum encounter distance $d_{\text {min }}$ differs from a Gaussian one, the mode of this distribution is $d_{\min }=0.27 \mathrm{pc}$, which is lower than the median value of $d_{\text {min }}$ given in Table 3 , while the distribution of encounter times is nearly Gaussian (Fig. 3b).

(4) Our simulations show that among the candidates considered, only the star GL 710 has a high probability of penetrating into the Oort cloud region.

\section{CONCLUSIONS}

Based on currently available space velocity data for about 35000 Hipparcos stars, we searched for stars closely encountering with the Solar system. For this purpose, we took stars within $30 \mathrm{pc}$ of the Sun and determined their orbits relative to the Sun based of the epicyclic approximation in the time interval from $-2 \mathrm{Myr}$ to $+2 \mathrm{Myr}$. We found nine new candidates in addition to the well-known list of such stars (Garcia-Sanchez et al. 2001). The use of the PCRV (Gontcharov 2006) and improved Hipparcos stellar parallaxes (Leeuwen 2007) made this possible.

As a result, we compiled the currently most complete (given the restrictions) list of Hipparcos stars that have close $(d<2$ pc) encounters with the Sun.

For the star GL 217.1, a well-known candidate for a passage close to the Sun, the new observational data were shown to change noticeably its previously known encounter parameters with the Sun. The encounter parameters found here are: $d_{\min }=1.28 \pm 0.06 \mathrm{pc}$ and $t_{\min }=-(861 \pm 40)$ thousand years. Improving the radial velocity for the white dwarf WD 0310-688 (HIP 14754) whose orbit passed at a distance $d_{\min }=1.61 \pm 0.19 \mathrm{pc}$ from the solar orbit about 300 thousand years ago is of current interest. Our statistical simulations showed that the star GL 710 has not only a high probability of penetrating into the Oort cloud, $P_{1}=0.86$, but also a nonzero probability, $P_{2}=1 \times 10^{-4}$, of penetrating into the region where the influence of the passing star on Kuiper Belt objects is significant.

\section{ACKNOWLEDGMENTS}

I wish to thank Prof. V.V. Orlov for a careful reading of the manuscript and his remarks and A.T. Bajkova for a discussion of the results. The SIMBAD searchable database was very helpful in the work. This study was supported by the Russian Foundation for Basic Research (project no. 08-02-00400) and in part by the "Origin and Evolution of Stars and Galaxies" Program of the Presidium of the Russian Academy of Sciences.

\section{REFERENCES}

Aannestad P.A., S.J. Kenyon, G.L. Hammon, et al., Astron. J. 105, 1033 (1993). 
Anosova J., V.V. Orlov, and N.A. Pavlova, Astron. Astrophys. 292, 115 (1994).

Bobylev V.V., A.T. Bajkova, and A.S. Stepanishchev, Astron. Lett. 34, 515 (2008).

Bobylev V.V., G.A. Gontcharov, and A.T. Bajkova, Astron. Rep. 50, 733 (2006).

Dehnen W., and J.J. Binney, MNRAS 298, 387 (1998).

Fuchs B., D. Breitschwerdt, M.A. Avilez, et al., MNRAS 373, 993 (2006).

Garcia-Sanchez J., R.A. Preston, D.L.Jones, et al., Astron. J. 117, 1042 (1999).

Garcia-Sanchez J., P.R.Weissman, R.A.Preston, et al., Astron. Astrophys. 379, 634 (2001).

Giampapa M.S., R. Rosner, V. Kashyap, et al., Astrophys. J. 463, 707 (1996).

Gontcharov G.A., Astron. Lett. 32, 759 (2006).

Greenstein J.L., and V.L. Trimble, Astrophys. J. 149, 283 (1967).

Hills J.G., Astron. J. 86, 1730 (1981).

The Hipparcos and Tycho Catalogues, ESA SP-1200 (1997).

Holmberg J., and C. Flinn, MNRAS 352, 440 (2004).

Holmberg J., B. Nordstrom, and J. Andersen, Astron. Astrophys. 475, 519 (2007).

Joshi Y.C., MNRAS 378, 768 (2007).

Kenyon S.J., and B. Bromley, Nature 432, 598 (2004).

Kharchenko N.V., R.-D. Scholz, A.E. Piskunov, et al., Astron. Nachr. 328, (2007).

van Leeuwen F., Astron. Astrophys. 474, 653 (2007).

Lindblad B., Arkiv Mat., Astron., Fysik A 20, (17), (1927).

Matthews R.A.J., R. Astron. Soc. Quart. J. 35,1 (1994).

Mullari A.A., and V.V. Orlov, Earth, Moon, and Planets (Kluwer Netherlands, 1996), Vol. 72, p. 19 .

Nordstrom B., M. Mayor, J. Andersen, et al., Astron. Astrophys. 419, 989 (2004).

Pauli E.M., R. Napiwotzki, U. Heber, et al., Astron. Astrophys. 447, 173 (2006).

Revina I.A., Analysis of the Motion of Celestial Bodies and Estimation of the Accuracy of Their Observations (Latvian Univ., Riga, 1988), p. 121.

Weissman P.R., Earth, Moon, and Planets (Kluwer, Netherlands, 1996), Vol. 72, p. 25.

Wilson R.E., General Catalogue of Stellar Radial Velocities (Carnegie Inst., Washington, DC, 1953), p. 601.

Translated by N. Samus' 
Table 1: Hipparcos stars within 30 pc of the Sun found here

\begin{tabular}{|r|l|r|r|r|r|r|r|}
\hline HIP & $\mathrm{SP}$ & $\begin{array}{r}d_{\text {min }}, \\
\mathrm{pc}\end{array}$ & $\begin{array}{r}t_{\text {min }}, \\
10^{3} \mathrm{yr}\end{array}$ & $\begin{array}{c}\pi \pm e_{\pi}, \\
\mathrm{mas}\end{array}$ & $\begin{array}{c}V_{r} \pm e_{V_{r}}, \\
\mathrm{~km} \mathrm{~s}^{-1}\end{array}$ & $\mathrm{n}$ & $\begin{array}{r}\varepsilon_{V_{r}}, \\
\mathrm{~km} \mathrm{~s}^{-1}\end{array}$ \\
\hline 1 & \multicolumn{1}{|c|}{2} & 3 & 4 & 5 & 6 & 7 & 8 \\
\hline 14754 & $\mathrm{DA}$ & 1.60 & -296 & $97.66 \pm 1.85$ & $33.8 \pm 3.2$ & 1 & \\
\hline 27887 & $\mathrm{~K} 3 \mathrm{~V}$ & 1.97 & -413 & $76.95 \pm 0.37$ & $30.7 \pm 0.3$ & 1 & \\
\hline $99461^{*}$ & $\mathrm{~K} 2 \mathrm{~V}$ & 2.05 & 41.1 & $166.25 \pm 0.27$ & $-129.0 \pm 0.2$ & 2 & 0.8 \\
\hline 24186 & $\mathrm{M} 0 \mathrm{~V}$ & 2.15 & -10.8 & $255.66 \pm 0.91$ & $244.4 \pm 0.2$ & 3 & 1.1 \\
\hline 105766 & $\mathrm{G} 5$ & 2.20 & 341 & $37.87 \pm 0.42$ & $-76.6 \pm 0.3$ & 2 & 0.2 \\
\hline 30344 & $\mathrm{~K} 0 \mathrm{~V}$ & 2.30 & -2035 & $34.10 \pm 0.62$ & $14.6 \pm 0.3$ & 1 & \\
\hline 26373 & $\mathrm{~K} 0 \mathrm{~V}$ & 2.33 & -762 & $39.82 \pm 1.36$ & $32.5 \pm 0.3$ & 1 & \\
\hline 104214 & $\mathrm{~K} 5 \mathrm{~V}$ & 2.79 & 18.6 & $286.82 \pm 6.78$ & $-65.9 \pm 0.1$ & 5 & 0.7 \\
\hline 104217 & $\mathrm{~K} 7 \mathrm{~V}$ & 2.80 & 19.6 & $285.88 \pm 0.54$ & $-64.3 \pm 0.1$ & 3 & 0.3 \\
\hline
\end{tabular}

Note. $n$ is the number of catalogs used to calculate the mean $V_{r} ; \varepsilon_{V_{r}}$ is the radial velocity error calculated from the external convergence (Gontcharov 2006); (*) HIP 99461 (GL 783) is present in the list by Mullari and Orlov (1996) but is absent in the lists by Garcia-Sanchez et al. (1999, 2001). 
Table 2: Data on the known Hipparcos stars from the solar neighborhood $30 \mathrm{pc}$ in radius encountering with the solar orbit within $d<2 \mathrm{pc}$

\begin{tabular}{|r|l|r|r|r|r|r|r|}
\hline HIP & & $\begin{array}{c}d_{\text {min }}, \\
\mathrm{pc}\end{array}$ & $\begin{array}{r}t_{\text {min }}, \\
10^{3} \mathrm{yr}\end{array}$ & $\begin{array}{c}\pi \pm e_{\pi}, \\
\mathrm{mas}\end{array}$ & $\begin{array}{c}V_{r} \pm e_{V_{r}}, \\
\mathrm{~km} \mathrm{~s}^{-1}\end{array}$ & $\mathrm{n}$ & $\begin{array}{c}\varepsilon_{V_{r}}, \\
\mathrm{~km} \mathrm{~s}^{-1}\end{array}$ \\
\hline 1 & \multicolumn{1}{c|}{3} & 4 & 5 & 6 & 7 & 8 \\
\hline 89825 & GL 710 & 0.21 & 1444 & $51.12 \pm 1.63$ & $-13.8 \pm 0.3$ & 4 & 8.1 \\
\hline 70890 & Proxima Cen & 0.89 & 27.4 & $771.64 \pm 2.60$ & $-25.1 \pm 0.9$ & & \\
\hline 71683 & $\alpha$ Cen A/B $*$ & 0.91 & 28.4 & $754.81 \pm 4.11$ & $-24.7 \pm 0.4$ & 1 & \\
\hline 57544 & AC+79 3888 & 1.06 & 46.0 & $186.86 \pm 1.70$ & $-111.6 \pm 0.2$ & 5 & 3.9 \\
\hline 87937 & Barnard's star & 1.15 & 9.8 & $548.31 \pm 1.51$ & $-110.6 \pm 0.2$ & 5 & 2.2 \\
\hline 27288 & GL 217.1 & 1.27 & -862 & $46.28 \pm 0.16$ & $24.7 \pm 1.1$ & 2 & 5.9 \\
\hline 54035 & Lalande 21185 & 1.43 & 20.5 & $392.64 \pm 0.67$ & $-85.8 \pm 0.1$ & 7 & 3.1 \\
\hline 26335 & GL 208 & 1.54 & -500 & $88.97 \pm 1.02$ & $22.0 \pm 0.2$ & 5 & 2.1 \\
\hline 57548 & Ross 128 & 1.92 & 72.4 & $298.04 \pm 2.30$ & $-31.0 \pm 0.2$ & 4 & 1.1 \\
\hline 110893 & GL 860 A & 1.93 & 90.0 & $249.94 \pm 1.87$ & $-34.0 \pm 0.1$ & 6 & 2.3 \\
\hline 92403 & Ross 154 & 1.96 & 157 & $336.72 \pm 2.03$ & $-10.7 \pm 0.2$ & 5 & 1.9 \\
\hline 38228 & HD 63433 & 2.02 & 1366 & $45.45 \pm 0.53$ & $-16.1 \pm 0.1$ & 6 & 1.2 \\
\hline
\end{tabular}

Note. $n$ is the number of catalogs used to calculate the mean $V_{r} ; \varepsilon_{V_{r}}$ is the radial velocity error calculated from the external convergence (Gontcharov 2006); $\left(^{*}\right)$ the encounter parameters were calculated from component A. 
Table 3: Hipparcos stars from the solar neighborhood $30 \mathrm{pc}$ in radius encountering with the solar orbit within $d<2$ pc

\begin{tabular}{|c|c|c|c|c|c|c|}
\hline $\begin{array}{c}\mathrm{HIP} \\
1 \\
\end{array}$ & 2 & $\begin{array}{c}\mathrm{SP} \\
3 \\
\end{array}$ & $\begin{array}{c}M / M_{\odot} \\
4\end{array}$ & $\begin{array}{c}\text { Ref } \\
5 \\
\end{array}$ & $\begin{array}{c}\bar{d}_{\text {min }}, 10^{3} \mathrm{yr} \\
6\end{array}$ & $\begin{array}{r}\bar{t}_{\text {min }}, \mathrm{pc} \\
7\end{array}$ \\
\hline 89825 & GL 710 & K7V & $0.4-0.6$ & (1) & $0.311 \pm 0.167$ & $1447 \pm 60$ \\
\hline 70890 & Proxima Cen & $\mathrm{M} 5 \mathrm{Ve}$ & $0.15 \pm 0.02$ & $(2)$ & $0.890 \pm 0.019$ & $27.4 \pm 0.1$ \\
\hline 71683 & $\alpha \operatorname{Cen~} \mathrm{A} / \mathrm{B}^{*}$ & G2V/K1V & $2.0 \pm 0.1$ & $(2)$ & $0.910 \pm 0.012$ & $28.4 \pm 0.1$ \\
\hline 57544 & $\mathrm{AC}+793888$ & M4 & $\approx 0.15$ & & $1.059 \pm 0.013$ & $46.0 \pm 0.3$ \\
\hline 87937 & Barnard's star & sdM4 & 0.144 & $(3)$ & $1.148 \pm 0.006$ & $9.8 \pm 0.1$ \\
\hline 27288 & GL 217.1 & A2Vann & 2.0 & $(1)$ & $1.275 \pm 0.064$ & $-861 \pm 40$ \\
\hline 54035 & Lalande 21185 & $\mathrm{M} 2 \mathrm{~V}$ & 0.39 & $(4)$ & $1.426 \pm 0.005$ & $20.5 \pm 0.1$ \\
\hline 26335 & GL 208 & $\mathrm{~K} 7$ & 0.47 & $(4)$ & $1.537 \pm 0.050$ & $-500 \pm 8$ \\
\hline 14754 & WD 0310-688 & $\overline{\mathrm{DA}}$ & $\approx 0.5$ & & $1.607 \pm 0.190$ & $-297 \pm 29$ \\
\hline 57548 & Ross 128 & $\mathrm{M} 4.5 \mathrm{~V}$ & 0.24 & $(4)$ & $1.920 \pm 0.028$ & $72.8 \pm 0.7$ \\
\hline 110893 & GL $860 \mathrm{~A}$ & M2V & 0.56 & (4) & $1.929 \pm 0.028$ & $90.3 \pm 0.5$ \\
\hline 92403 & Ross 154 & $\mathrm{M} 3.5 \mathrm{Ve}$ & 0.23 & $(4)$ & $1.959 \pm 0.030$ & $157 \pm 1$ \\
\hline 27887 & HD 40307 & K3V & $0.75_{-0.71}^{+0.78}$ & $(5)$ & $1.974 \pm 0.035$ & $-413 \pm 5$ \\
\hline 38228 & HD 63433 & G5IV & $0.88_{-0.84}^{+0.98}$ & $(5)$ & $2.038 \pm 0.099$ & $1366 \pm 20$ \\
\hline
\end{tabular}

Note. The stellar mass estimates were taken from the following papers: (1) Garcia-Sanchez et al. (1999); Anosova et al. (1994); (3) Giampapa et al. (1996); (4) Mullari and Orlov (1996); and (5) Holmberg et al. (2007). If there is no reference, then a typical mass is given; $(*)$ the sum of the masses is given. 


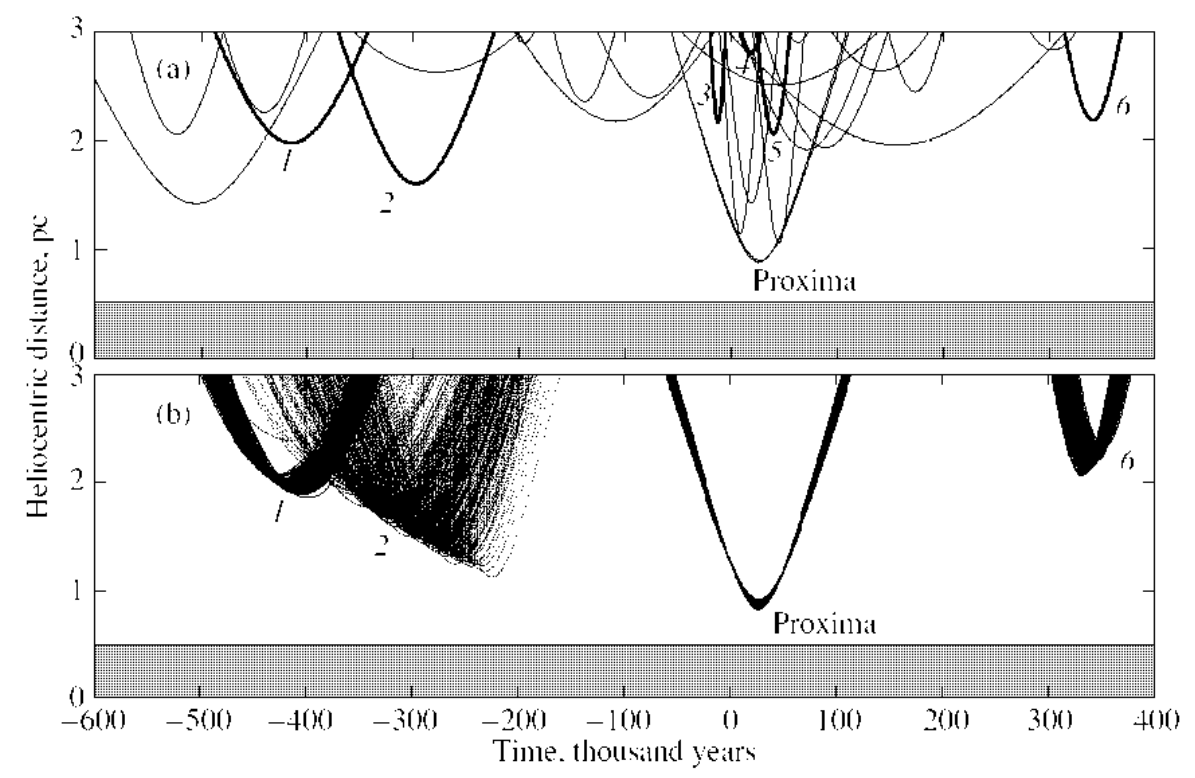

Fig. 1. (a) Stellar trajectories relative to the Sun. The trajectories of the stars found here are highlighted by the thick lines: HIP 27887 (1), HIP 14754 (2), HIP 24186 (3), the pair of HIP 104214 and HIP 104217 (4), HIP 99461 (5), and HIP 105766 (6). (b) The model trajectories of four stars computed by taking into account the random errors in the observational data (300 realizations). The trajectories hatch the $3 \sigma$ confidence regions; the Oort cloud region is shaded.

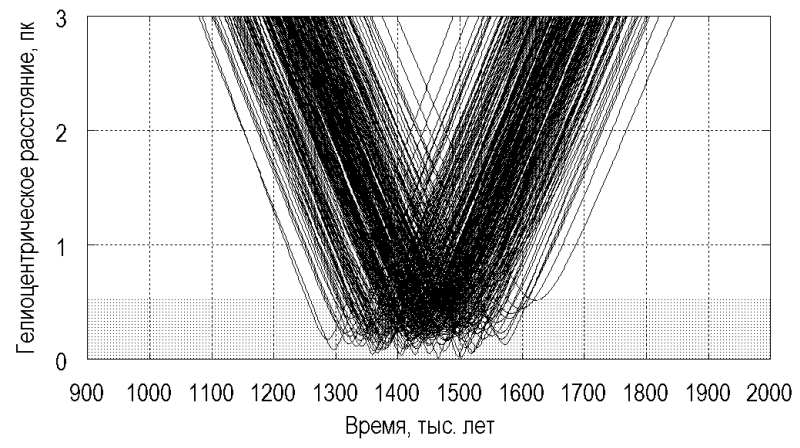

Fig. 2. Model trajectories of the star HIP 89825 (GL 710) relative to the Sun computed by taking into account the random errors in the observational data (300 realizations). The trajectories hatch the $3 \sigma$ confidence region; the Oort cloud region is shaded. 

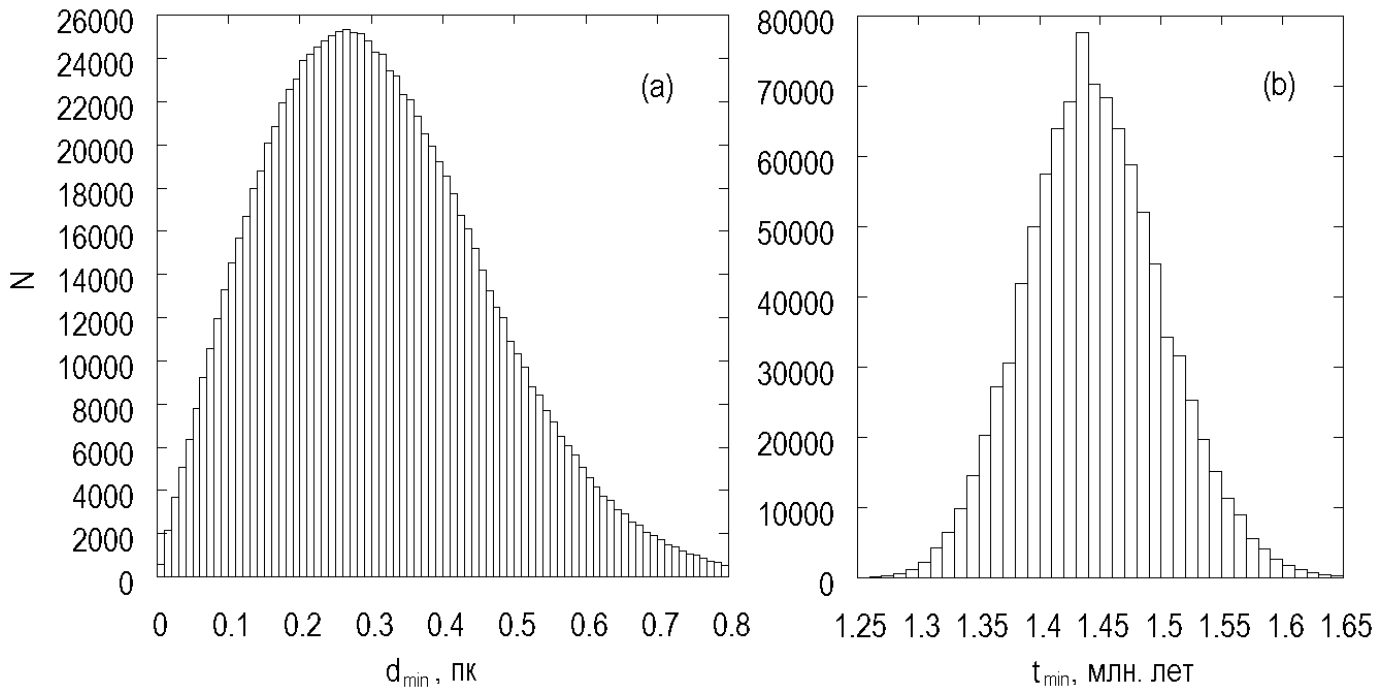

Fig. 3. Model encounter parameters of the star HIP 89825 (GL 710) with the Sun. (a) The distribution of minimum distance $d_{m i n}$; (b) the histogram of encounter times $t_{\text {min }}$. 